\title{
Few-Body Universality in Halo Nuclei
}

\author{
H.-W. Hammer ${ }^{1,2, a}$ \\ ${ }^{1}$ Institut für Kernphysik, Technische Universität Darmstadt, 64289 Darmstadt, Germany \\ ${ }^{2}$ ExtreMe Matter Institute EMMI, GSI Helmholtzzentrum für Schwerionenforschung GmbH, 64291 Darm- \\ stadt, Germany
}

\begin{abstract}
Few-body systems with resonant S-wave interactions show universal properties which are independent of the interaction at short distances. These properties include a geometric spectrum of three- and higher-body bound states and universal correlations between few-body observables. They can be observed on a wide range of scales from hadrons and nuclei to ultracold atoms. In this contribution, we focus on few-body universality in halo nuclei which can be considered as effective few-body systems consisting of halo nucleons and a core. This concept provides a unifying framework for halo nuclei with calculable corrections. Recent progress in this field with an emphasis on the possibility of finding Efimov states in halo nuclei is discussed.
\end{abstract}

\section{Introduction}

Particles with resonant interactions have universal properties that are independent of their interaction at short distances. The scattering of particles with short-range interactions at sufficiently low energy is determined by their S-wave scattering length $a$. Let us consider equal mass bosons of mass $m$ for simplicity. If $a$ is much larger than the range of the interaction $R$, the system shows universal properties [1]. The simplest example is the existence of a shallow two-body bound state with binding energy

$$
B_{2}=\frac{1}{m a^{2}}+O(R / a),
$$

and mean-square separation $a^{2} / 2$ if $a$ is large and positive. ${ }^{1}$

If a third particle is added, a three-body parameter $\kappa_{*}$ is required to fully characterize the universal properties. For fixed scattering length, this implies universal correlations between different threebody observables such as the Phillips line [2]. Moreover, the Efimov effect [3] generates a universal spectrum of three-body bound states with binding energy

$$
B_{3}=-\frac{1}{m a^{2}}+\left(e^{-2 \pi / s_{0}}\right)^{n-n_{*}} \exp \left[\Delta(\xi) / s_{0}\right] \frac{\kappa_{*}^{2}}{m}+O(R / a),
$$

where the angle $\xi$ is defined by $\tan \xi=-\left(m B_{3}\right)^{1 / 2} a$, the index $n$ labels the three-body states, $\Delta(\xi)$ is a universal function with $\Delta(-\pi / 2)=0$, and $s_{0}=1.00624 \ldots$ is a transcendental number (see Ref. [4] for more details). This spectrum is illustrated in Fig. 1 in the two-dimensional plane spanned by the

\footnotetext{
a e-mail: Hans-Werner.Hammer@physik.tu-darmstadt.de

${ }^{1}$ We use natural units with $\hbar=c=1$.
} 


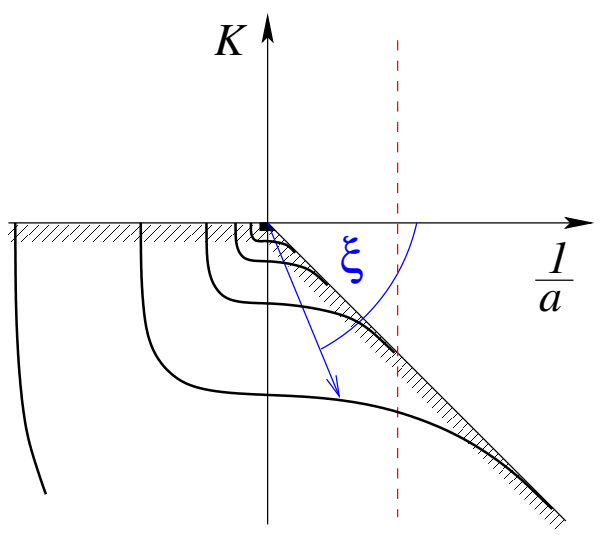

Figure 1. Illustration of the Efimov spectrum: The energy variable $K=\operatorname{sgn}(E) \sqrt{m|E|}$ is shown as a function of the inverse scattering length $1 / a$. The solid lines indicate the Efimov states while the hashed areas give the scattering thresholds. The dashed vertical line illustrates an exemplary system with fixed scattering length.

energy variable $K=\operatorname{sgn}(E) \sqrt{m|E|}$ and the inverse scattering length $1 / a$. The solid lines indicate the Efimov states while the hashed areas give the scattering thresholds below which the bound states can exist. The dashed vertical line illustrates an exemplary system with fixed scattering length.

In the unitary limit $1 / a=0$, Eq. (2) reduces to the geometric spectrum

$$
B_{3}=\left(e^{-2 \pi / s_{0}}\right)^{n-n_{*}} \frac{\kappa_{*}^{2}}{m}+O(R / a) .
$$

It is now evident that the three-body parameter $\kappa_{*}$ is chosen as the binding momentum of the state with label $n_{*}$, but other definitions are possible.

The spectrum shown in Fig. 1 is invariant under discrete scaling transformations with the preferred scaling factor $\lambda_{0}=e^{\pi / s_{0}}$ :

$$
\kappa_{*} \quad \longrightarrow \quad \kappa_{*}, \quad a \longrightarrow \lambda_{0}^{n} a, \quad B_{3} \longrightarrow \lambda_{0}^{-2 n} B_{3},
$$

where $n$ is any integer. This discrete scale invariance holds for all few-body observables and is a clear signature of a renormalization group limit cycle in the three-body system [5]. Its manifestation in observables is often referred to as Efimov physics.

If more particles are added, no new parameters are needed for renormalization at leading order [6]. As a consequence, all four-body observables in the universal regime are governed by the same limit cycle and can be characterized by $a$ and $\kappa_{*}$. A similar behavior is expected for higher-body observables. In ultracold atoms, these properties have now been verified for up to five particles [7, 8]. For a review of the status in nuclear and particle physics, see Ref. [9].

\section{Halo EFT}

Here, we use this universality as the starting point for an effective field theory (EFT) expansion for strongly-interacting quantum systems with short-range interactions. This universal EFT is applicable to any system with resonant interactions, i.e., any system with short-range interactions and large scattering lengths. Examples include nuclear halo states, ultracold atoms close to a Feshbach resonance, and hadronic molecules in particle physics. The breakdown scale $M_{\text {high }}$ of this theory is set 
by the lowest energy degree-of-freedom not explicitly included in the theory. In nuclear and particle physics, this is typically given by one-pion exchange such that $M_{\text {high }} \sim m_{\pi}$. In ultracold atoms, $M_{\text {high }}$ is determined by the scale set by the van der Waals interaction. The typical momentum scale of the theory is $M_{\text {low }} \sim 1 / a \sim k$. For momenta $k$ of the order of the breakdown scale $M_{\text {high }}$ or above, the omitted short-range physics is resolved and has to be treated explicitly. This framework exploits the appearance of a large scattering length $a \gg 1 / M_{\text {high }}$, independent of the mechanism generating it.

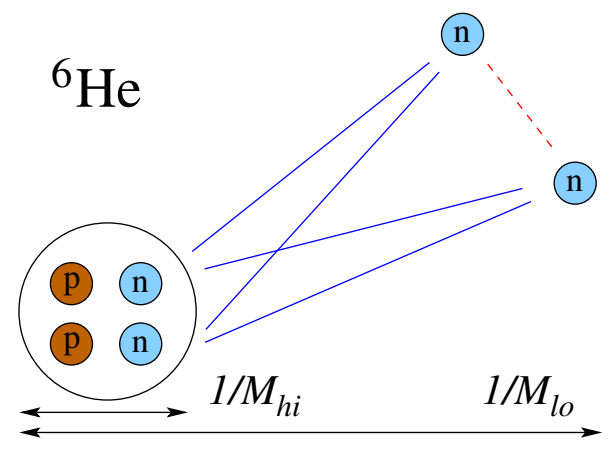

Figure 2. Illustration of the antisymmetrization of neutrons in the halo nucleus ${ }^{6} \mathrm{He}$ treated as $\alpha+n+n$ in the framework of halo EFT. The exchange illustrated by the dashed line involves active degrees of freedom and is antisymmetric, while the exchanges indicated by the solid lines are not included.

The application of these ideas to nuclear physics has led to the development of the so-called pionless EFT, which is applicable for typical momenta below the pion mass. (See Refs. [10-12] for reviews and references to earlier work.) If one also introduces field operators for certain tightly-bound clusters of nucleons, such as $\alpha$ particles, the pionless EFT is usually referred to as halo EFT [13, 14]. This approach can be viewed as a generalization of nuclear cluster models. One might worry about the missing antisymmetrization of nucleons in the core, which are not active degrees of freedom in halo EFT, and the halo nucleons (see Fig. 2 for an illustration in the case of ${ }^{6} \mathrm{He}$ treated as $\alpha+n+n$.). However, the effect of exchanging nucleons in the core with halo nucleons on observables is governed by the same expansion in $M_{\text {low }} / M_{\text {high }}$ as the EFT itself. Therefore, these effects are controlled and remain small in its applicability domain.

Halo EFT complements ab initio approaches to halo nuclei by providing universal relations between different few-body observables. These relations can be combined with input from ab initio theory or experiment to predict halo properties. Halo EFT has recently been applied to describe the structure and reactions of many known and predicted one- and two-neutron halo systems. For oneneutron halos it essentially reproduces the effective range expansion, but their electromagnetic structure and reactions can be predicted [15-20]. Moreover, the natural accuracy limits of cluster models can be estimated from the order at which local gauge invariant couplings to the currents appear.

The Efimov effect provides a natural binding mechanism for two-neutron halos, but the contribution of higher partial waves and partial wave mixing complicate the situation compared to ultracold atoms. While halo EFT naturally accommodates resonant interactions in higher partial waves $[13,14]$, there is no Efimov effect in this case $[21,22]$. The possibility of observing excited Efimov states in two-neutron halo nuclei was investigated in Refs. [23-25]. The two-neutron halo nucleus ${ }^{6} \mathrm{He}$ is particularly interesting due to the dominance of $\mathrm{P}$-wave neutron- $\alpha$ interactions. Its structure and renormalization properties were recently investigated in Refs. [26, 27]. Finally, the electromag- 
netic structure and breakup of two-neutron halo nuclei has also been calculated in halo EFT [28-30]. See Ref. [31] for a discussion of the Coulomb dissociation of ${ }^{11} \mathrm{Li}$ at this conference.

Proton halo systems are less common due to the delicate interplay between attraction from the strong interaction and the repulsion from the Coulomb interaction. The presence of the Coulomb barrier introduces the Coulomb momentum as a new scale into the problem, which is given by the inverse of the Bohr radius of the system. The additional Coulomb scale complicates the power counting since it is independent of the hadronic scales. Higa et al. [32], e.g., treated the Coulomb momentum as a high-momentum scale in their study of $\alpha-\alpha$ scattering. However, this treatment is not always appropriate. The correct scaling of the Coulomb momentum depends on the system considered. This is one of the issues investigated in recent studies of proton halo nuclei and their electromagnetic structure and reactions [33-38]. For a discussion of radiative proton capture on ${ }^{7} \mathrm{Be}$ at this conference, see Ref. [39]. Finally, halo nuclei containing strange baryons are also amenable to halo EFT [40-43], but the scarcity of data in this sector presents a particular challenge.

In the remainder of this proceedings contribution, we review some of the calculations discussed above in more detail. A special emphasis is placed on the possibility of finding Efimov states in halo nuclei.

\section{Excited Efimov states in halo nuclei}

As discussed above, the Efimov effect provides a natural binding mechanism for two-neutron halo nuclei. Thus the universal EFT, which is based on an expansion around the Efimov limit, becomes a powerful tool to describe their properties. Since the strength of the interaction between the neutrons and the core is fixed, however, the identification of Efimov physics is more delicate than for ultracold atoms. In particular, the log-periodic dependence of observables on the scattering length can not be used to identify Efimov physics. Instead one looks for excited states which (approximately) satisfy the universal scaling relation for bound states encapsulated in Eq. (2).

Halo nuclei have been discussed as possible candidates for Efimov states since more than 30 years [44]. It is particularly instructive to plot the candidate nuclei in a two-dimensional plane characterized by the neutron-core energy $E_{n n}$ and neutron-neutron energy $E_{n n}$ in units of the three-body ground state energy $E_{g s}$ introduced by Amorim et al. [45]. If a given nucleus lies within a certain boundary curve that weakly depends on the mass number $A$ of the core, it should display an excited Efimov state if the ground state is bound by the Efimov effect. The status for ${ }^{11} \mathrm{Li},{ }^{12} \mathrm{Be},{ }^{14} \mathrm{Be},{ }^{18} \mathrm{C}$, and ${ }^{20} \mathrm{C}$ according to a recent EFT analysis [23] assuming that these nuclei can be treated as S-wave two-neutron halo nuclei at leading order is summarized in Fig. 3. Note that the point for ${ }^{20} \mathrm{C}$ has shifted as indicated by the arrow from using the neutron separation energies of the recent AME2012 atomic mass evaluation [46] instead of the older values used in Ref. [23]. As a consequence, none of the depicted nuclei are expected to exhibit an excited Efimov state.

The most promising system known so far is the ${ }^{22} \mathrm{C}$ halo nucleus which was found to display an extremely large matter radius [47] and has a significant S-wave component in the $n-{ }^{20} \mathrm{C}$ system [48]. Acharya et al. [24] used halo EFT to explore the correlation between the $n^{20} \mathrm{C}$ energy and the twoneutron separation energy of ${ }^{22} \mathrm{C}$. They showed that an excited Efimov state in ${ }^{22} \mathrm{C}$ is excluded by combining this correlation with the matter radius measurement of Tanaka et al. [47]. See Ref. [31] for a more detailed discussion of the work at this conference.

Whether heavier two-neutron halos exist is still an open question, although there is some experimental evidence that the ground states of ${ }^{31} \mathrm{Ne}$ and ${ }^{37} \mathrm{Mg}$ have a low one-neutron separation energy and are deformed P-wave halos $[49,50]$. This makes it worthwhile to investigate the possibility for Efimov states in heavier nuclei. 


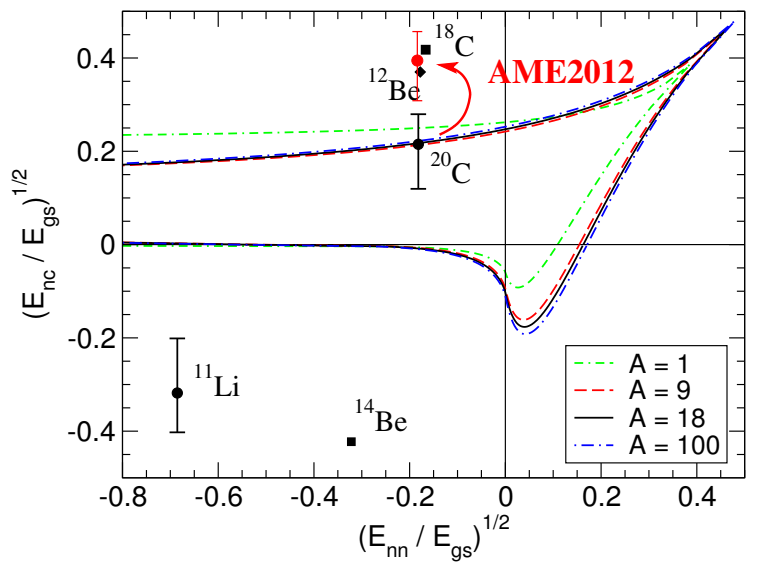

Figure 3. Boundary curves for the existence of an excited Efimov state as function of the neutron-core energy $E_{n n}$ and neutron-neutron energy $E_{n n}$ in units of the three-body ground state energy $E_{g s}$. The boundary curves depend weakly on the core mass $A$. The shift of ${ }^{20} \mathrm{C}$ from using the values of the newer AME2012 atomic mass evaluation [46] is indicated by the arrow.

There has been much interest recently, both experimentally and theoretically, in determining precise values for masses, understanding shell evolution and the location of the dripline in the neutron rich calcium isotopes [51-56]. Coupled-cluster calculations of neutron rich calcium isotopes that included coupling to the scattering continuum and schematic three-nucleon forces, suggested that there is an inversion of the $g d s$ shell-model orbitals in ${ }^{53,55,61} \mathrm{Ca}$. In particular it was suggested that a large $\mathrm{S}$-wave scattering length might occur in ${ }^{61} \mathrm{Ca}$ with interesting implications for ${ }^{62} \mathrm{Ca}$.

Hagen and collaborators [25] used the coupled-cluster method combined with modern chiral effective theory interactions and follow the method outlined in Ref. [57] to compute the elastic scattering of neutrons on ${ }^{60} \mathrm{Ca}$. They analyzed the resulting phase shift data to obtain quantitative estimates for the scattering length and the effective range and showed that a large scattering length can be expected in this system. The results obtained from the ab initio calculations were then used as input for halo EFT. They analyzed the implications of the coupled cluster results for the ${ }^{60} \mathrm{Ca}-n-n$ system. Specifically, they focused on the signals of Efimov physics that are a consequence of the large scattering length in the ${ }^{60} \mathrm{Ca}-n$ and $n-n$ systems.

This is illustrated in Fig. 4, where the universal correlation between the ${ }^{61} \mathrm{Ca}-n$ scattering length and the two-neutron separation energy of ${ }^{62} \mathrm{Ca}$ is shown. For ${ }^{62} \mathrm{Ca}$, the discrete scaling factor governing the energy spectrum is approximately $16^{2}=256$ [1], which is slightly more favorable than in the case of three identical bosons. The asymptotic scaling ratio applies for deep states and in the unitary limit of infinite scattering length. For levels near threshold, however, the ratio of energies can be significantly smaller (see Fig. 1 and the corresponding discussion in Ref. [1]). In the case of ${ }^{62} \mathrm{Ca}$, the whole energy region between $S_{n} \approx 5-8 \mathrm{keV}$ and the breakdown scale $S_{\text {deep }} \approx 500 \mathrm{keV}$ is available for Efimov states. In the shaded region of Fig. 4 halo EFT breaks down. Around a two-neutron separation energy of about $230 \mathrm{keV}$ indicated by the dashed vertical line, the ${ }^{60} \mathrm{Ca}-n$ scattering length jumps from plus to minus infinity and an excited Efimov state appears. It is thus conceivable that ${ }^{62} \mathrm{Ca}$ would display an excited Efimov state and unlikely that it would not display any Efimov states at all. 


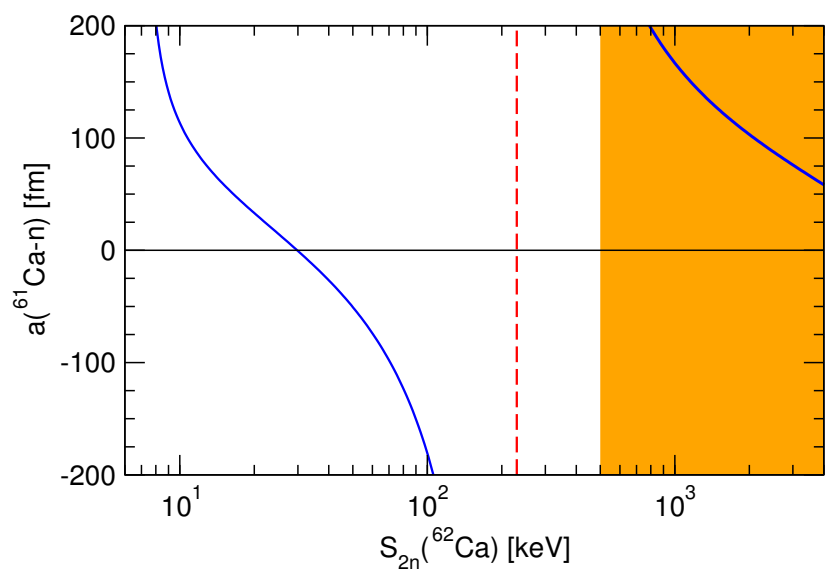

Figure 4. The correlation between the ${ }^{61} \mathrm{Ca}-n$ scattering length and the two-neutron separation energy $S_{2 n}$ of ${ }^{62} \mathrm{Ca}$. The emergence of an excited Efimov state around $S_{2 n}=230 \mathrm{keV}$ is indicated by the vertical dashed line. In the shaded region, halo EFT breaks down.

\section{Conclusion}

In this proceedings contribution, we have reviewed the manifestations of few-body universality in halo nuclei with a special focus on the possibility of observing Efimov states. While the ground state of many S-wave halo nuclei is close to the Efimov limit, there is currently no observed halo nucleus that displays an excited Efimov state or is likely to display such a state. There is some theoretical evidence that the situation might be different for ${ }^{62} \mathrm{Ca}$. The results of Hagen et al. [25] imply that ${ }^{62} \mathrm{Ca}$ is possibly the largest and heaviest halo nucleus in the chart of nuclei. They showed that as a result a large number of observables would display characteristic features of Efimov physics. Measurements of these observables clearly pose a significant challenge for experiment. For example, ${ }^{58} \mathrm{Ca}$ is the heaviest Calcium isotope that has been observed experimentally [58]. However, future radioactive beam facilities might provide access to calcium isotopes as heavy as ${ }^{68} \mathrm{Ca}$ and thereby facilitate a test of this hypothesis.

Another important aspect from few-body universality in halo nuclei beyond Efimov physics is the possibility to formulate an effective field theory exploiting the universal aspects of the resonant interactions involved. This effective field theory is called halo EFT. It is complementary to ab initio approaches to halo nuclei by providing universal relations between different few-body observables. These relations can be combined with input from ab initio theory or experiment to predict halo properties. Moreover, they allow to test the consistency of different approaches and/or experiments. A particular strength of this approach lies in the possibility to describe the electroweak structure and reactions of halo nuclei in a model independent way with controlled error estimates. A brief overview of the activities in this exciting area with a large future potential was given in Section 2 and will not be repeated here.

\section{Acknowledgements}

This work was supported by the BMBF under contract 05P12PDFTE and by the Helmholtz Association under contract HA216/EMMI. Collaborations and discussions on this topic with B. Acharya, D. Canham, C. Forssén, G. Hagen, P. Hagen, D. Phillips, L. Platter, and E. Ryberg are gratefully acknowledged. 


\section{References}

[1] E. Braaten and H.-W. Hammer, Phys. Rept. 428, 259 (2006) [cond-mat/0410417].

[2] A. C. Phillips, Nucl. Phys. A 107, 209 (1968).

[3] V. Efimov, Phys. Lett. B 33, 563 (1970).

[4] E. Braaten, H.-W. Hammer, and M. Kusunoki, Phys. Rev. A 67, 022505 (2003) [condmat/0201281].

[5] P.F. Bedaque, H.-W. Hammer, and U. van Kolck, Phys. Rev. Lett. 82, 463 (1999) [nuclth/9809025].

[6] L. Platter, H.-W. Hammer, and U. G. Meißner, Phys. Rev. A 70, 052101 (2004) [condmat/0404313].

[7] F. Ferlaino, R. Grimm, Physics 3, 9 (2010).

[8] A. Zenesini et al., New J. Phys. 15, 043040 (2013) [arXiv:1205.1921].

[9] H.-W. Hammer and L. Platter, Ann. Rev. Nucl. Part. Sci. 60, 207 (2010) [arXiv:1001.1981 [nuclth]].

[10] S. R. Beane, P. F. Bedaque, W. C. Haxton, D. R. Phillips, and M. J. Savage, At the frontier of particle physics, Vol. 1, M. Shifman (ed.) (World Scientific, Singapore, 2001) 133 [nucl-th/0008064].

[11] P. F. Bedaque and U. van Kolck, Ann. Rev. Nucl. Part. Sci. 52, 339 (2002) [nucl-th/0203055].

[12] E. Epelbaum, H.-W. Hammer, and U.-G. Meißner, Rev. Mod. Phys. 81, 1773 (2009) [arXiv:0811.1338 [nucl-th]].

[13] C. A. Bertulani, H.-W. Hammer, and U. van Kolck, Nucl. Phys. A 712, 37 (2002) [nuclth/0205063].

[14] P. F. Bedaque, H.-W. Hammer, and U. van Kolck, Phys. Lett. B 569, 159 (2003) [nuclth/0304007].

[15] H.-W. Hammer and D. R. Phillips, Nucl. Phys. A 865, 17 (2011) [arXiv:1103.1087 [nucl-th]].

[16] G. Rupak and R. Higa, Phys. Rev. Lett. 106, 222501 (2011) [arXiv:1101.0207 [nucl-th]].

[17] L. Fernando, R. Higa, and G. Rupak, Eur. Phys. J. A 48, 24 (2012) [arXiv:1109.1876 [nucl-th]].

[18] G. Rupak, L. Fernando, and A. Vaghani, Phys. Rev. C 86, 044608 (2012) [arXiv:1204.4408 [nucl-th]].

[19] B. Acharya and D. R. Phillips, Nucl. Phys. A 913, 103 (2013) [arXiv:1302.4762 [nucl-th]].

[20] X. Zhang, K. M. Nollett, and D. R. Phillips, Phys. Rev. C 89, 024613 (2014) [arXiv:1311.6822 [nucl-th]].

[21] Y. Nishida, Phys. Rev. A 86, 012710 (2012) [arXiv:1111.6961 [cond-mat.quant-gas]].

[22] E. Braaten, P. Hagen, H.-W. Hammer, and L. Platter, Phys. Rev. A 86, 012711 (2012) [arXiv:1110.6829 [cond-mat.quant-gas]].

[23] D. L. Canham and H.-W. Hammer, Eur. Phys. J. A 37, 367 (2008) [arXiv:0807.3258 [nucl-th]].

[24] B. Acharya, C. Ji, and D. R. Phillips, Phys. Lett. B 723, 196 (2013) [arXiv:1303.6720 [nucl-th]].

[25] G. Hagen, P. Hagen, H.-W. Hammer, and L. Platter, Phys. Rev. Lett. 111, 132501 (2013) [arXiv:1306.3661 [nucl-th]].

[26] J. Rotureau and U. van Kolck, Few Body Syst. 54, 725 (2013) [arXiv:1201.3351 [nucl-th]].

[27] C. Ji, C. Elster, and D. R. Phillips, Phys. Rev. C 90, 044004 (2014) [arXiv:1405.2394 [nucl-th]].

[28] P. Hagen, H.-W. Hammer, and L. Platter, Eur. Phys. J. A 49, 118 (2013) [arXiv:1304.6516 [nuclth]].

[29] P. Hagen, Effective Field Theory for Halo Nuclei, Dissertation, University of Bonn (2014), http://hss.ulb.uni-bonn.de/2014/3526/3526.htm . 
[30] B. Acharya, Properties of One- and Two-Nucleon Halo Nulcei in Effective Field Theory, $\mathrm{PhD}$ thesis, Ohio University (2015), http://rave.ohiolink.edu/etdc/view?acc_num=ohiou1423835581 .

[31] B. Acharya and D. Phillips, these proceedings [arXiv:1508.02697].

[32] R. Higa, H.-W. Hammer, and U. van Kolck, Nucl. Phys. A 809, 171 (2008) [arXiv:0802.3426 [nucl-th]].

[33] R. Higa, EPJ Web Conf. 3, 06001 (2010) [arXiv:1001.0540 [nucl-th]].

[34] E. Ryberg, C. Forssén, H.-W. Hammer, and L. Platter, Phys. Rev. C 89, 014325 (2014) [arXiv:1308.5975 [nucl-th]].

[35] X. Zhang, K. M. Nollett, and D. R. Phillips, Phys. Rev. C 89, 051602 (2014) [arXiv:1401.4482 [nucl-th]].

[36] E. Ryberg, C. Forssén, H.-W. Hammer, and L. Platter, Eur. Phys. J. A 50, 170 (2014) [arXiv:1406.6908 [nucl-th]].

[37] X. Zhang, K. M. Nollett, and D. R. Phillips, arXiv:1507.07239 [nucl-th].

[38] E. Ryberg, C. Forssén, H.-W. Hammer, and L. Platter, arXiv:1507.08675 [nucl-th].

[39] X. Zhang, K.M. Nollet, D.R. Phillips, these proceedings [arXiv:1508.06935].

[40] H.-W. Hammer, Nucl. Phys. A 705, 173 (2002) [nucl-th/0110031].

[41] S. I. Ando, G. S. Yang, and Y. Oh, Phys. Rev. C 89, 014318 (2014) [arXiv:1310.1432 [nucl-th]].

[42] S. I. Ando and Y. Oh, Phys. Rev. C 90, 037301 (2014) [arXiv:1407.1608 [nucl-th]].

[43] S. I. Ando, U. Raha, and Y. Oh, Phys. Rev. C 92, 024325 (2015) [arXiv:1507.01260 [nucl-th]].

[44] D.V. Fedorov, A.S. Jensen, and K. Riisager, Phys. Rev. Lett. 73, 2817 (1994).

[45] A. E. A. Amorim, T. Frederico, and L. Tomio, Phys. Rev. C 56, R2378 (1997) [nucl-th/9708023].

[46] M. Wang et al., Chin. Phys. C 36, 1603 (2012).

[47] K. Tanaka et al., Phys. Rev. Lett. 104, 062701 (2010).

[48] W. Horiuchi and Y. Suzuki, Phys. Rev. C 74, 034311 (2006).

[49] T. Nakamura et al., Phys. Rev. Lett. 112, 142501 (2014).

[50] N. Kobayashi et al., Phys. Rev. Lett. 112, 242501 (2014).

[51] A. Lapierre et al., Phys. Rev. C 85, 024317 (2012).

[52] A.T. Gallant et al., Phys. Rev. Lett. 109, 032506 (2012).

[53] J. D. Holt and A. Schwenk, J. Phys. G 39, 085111 (2012).

[54] G. Hagen, M. Hjorth-Jensen, G.R. Jansen, R. Machleidt, and T. Papenbrock, Phys. Rev. Lett. 109, 032502 (2012).

[55] W. Nazarewicz et al., Phys. Rev. C 53, 740 (1996).

[56] J. Erler et al., Nature 486, 509 (2012).

[57] G. Hagen and N. Michel, Phys. Rev. C 86, 021602(R) (2012).

[58] O. B. Tarasov et al., Phys. Rev. Lett. 102, 142501 (2009). 